\title{
PREFACE
}

\section{Affective Communication: Characteristics, Disorders, and Clinical Implications}

\begin{abstract}
The purpose of this issue of Seminars in Speech and Language is to delineate communication of emotion with an emphasis on the affective behaviors displayed and perceived during communication interaction. Affective communication disorders, that is, deficits in expressing or perceiving messages that relate emotional information, occur in adults with various forms of brain damage and in children with developmental and neurological disorders. Communication of affect is conveyed through verbal and nonverbal modalities and each of these modalities can independently relay information (e.g., the voice alone can "tell" a listener how a speaker is feeling) or in combination relay information (e.g., sarcasm is conveyed when words and nonverbal cues seem discordant or ambiguous). When an individual has difficulty expressing or perceiving how a communicative partner is feeling, interpersonal relationships and social competence are jeopardized. Affective communication has received attention in the scientific realm where affective speech prosody, facial expression, and higher-level language competencies such as humor and inferencing have been studied. Clinicians regularly work with patients who may display such affective disorders as flat affect, social inappropriateness, and emotional lability, and yet many lack an understanding of what might cause these behaviors and how to assess and manage them to maximize communicative success.

Results from investigations of affective communication and its disorders have not yielded widely accepted theories of affect perception and
\end{abstract}

production. To date it is not known if there is a central processor that regulates and maintains systems responsible for affective communication, or if the linguistic and perceptual modalities used to communicate affect are separate and governed independently. The articles in this issue were brought together to provide readers with a basic understanding of affect and affective communication disorders with consideration for the relevant theories that have been proposed. The first article, written by Karow and Connors, provides a definition of affect and an overview of the verbal and nonverbal modalities of normal affective communication, followed by disorders of affective communication and regulation. They discuss in detail the modality of facial expression and provide tools for assessing affect communication.

Next, Sidtis and Van Lancker Sidtis consider the role of affective prosody in the more global scheme of prosodic disorders and propose a neurobehavior-based approach to guide researchers and clinicians. The third article is dedicated to the verbal modalities of affective communication. Lehman Blake discusses affective language and humor appreciation after right hemisphere brain damage. She also provides clinical assessment and treatment guidelines, which may also apply to patients with traumatic brain injury.

Although this issue is primarily dedicated to adult populations, the fourth article, written by Timler, is dedicated to children's emotion comprehension as expressed through facial expressions and situational cues. Timler also pro-

Affective Communication: Characteristics, Disorders, and Clinical Implications. Editors in Chief, Nancy HelmEstabrooks, Sc.D., and Nan Bernstein Ratner, Ed.D.; Guest Editor, Colleen M. Karow, Ph.D. Seminars in Speech and Language, volume 24, number 2, 2003. ${ }^{1}$ Department of Communicative Disorders, University of Rhode Island, Kingston, Rhode Island. Copyright (C) 2003 by Thieme Medical Publishers, Inc., 333 Seventh Avenue, New York, NY 10001, USA. Tel: +1(212) 584-4662. 0734-0478,p;2003,24;02,067,068,ftx,en;ss100150b. 
vides suggestions for assessment and intervention of children's emotion comprehension skills.

In the final article, Auerbach and Karow provide a description of the neurobehavioral assessment of mood and affective disorders in normal and neurologically impaired patients. They explain how neurological deficits may limit the capacity of the individual to communicate mood and feelings and describe how mood, sleep, and other psychiatric disorders can mimic affective deficits.

A fundamental understanding of affect and mood is of relevance to speech-language pathologists and other practitioners who work with patients with neurogenic cognitive and communication disorders. Emotion changes can occur as a result of many different types of neurological diseases. The articles in this issue focus primarily on those changes that have a direct impact on communication and social interaction. It is hoped that these will spur a more active interest by clinicians in the exploration of clinical practices that dovetail with emerging theories of affective communication.

Colleen M. Karow, Ph.D. Guest Editor ${ }^{1}$ 